\title{
Competence in pulmonary endoscopy emergencies
}

\author{
Claudio F. SIMONASSI ${ }^{1}$ *, Maria MAJORI ${ }^{2}$, Maria G. COVESNON ${ }^{1}$, Annalisa BRIANTI ${ }^{1}$, \\ Luigi LAZZARI AGLI ${ }^{3}$, Eleonora MEONI ${ }^{4}$, Antonella IELPO 5, Lorenzo CORBETTA 6
}

1 Unit of Pulmonology, Villa Scassi Hospital, Genoa, Italy; ${ }^{2}$ Unit of Pulmonology, Thoracic Endoscopy, University Hospital of Parma, Parma, Italy; ${ }^{3}$ Unit of Pulmonology, AUSL Romagna, Rimini, Italy; ${ }^{4}$ Emergency Unit, SS Cosma e Diamiano Hospital Pescia, Pistoia, Italy; ${ }^{5}$ University of Parma, Parma, Italy; ${ }^{6}$ Unit of Interventional Pulmonology, University Hospital of Careggi, Florence, Italy

*Corresponding author: Claudio F. Simonassi, S.C. Pneumologia Ospedale Villa Scassi Pad.9/5, Corso Onofrio Scassi 1,16149 Genoa, Italy.

E-mail: claudiofrancesco.simonassi@asl3.liguria.it

\section{A B S T RA CT}

In clinical practice, interventional pulmonologists face several situations which can lead to dramatic consequences especially regarding ventilation and require immediate intervention. We describe the main pathological conditions where an urgent bronchoscopy is crucial because they act through mechanisms such as airway obstructions or alteration of the anatomic integrity of the tracheobronchial tree. We point out the problems resulting from inhalation of foreign bodies, one of the most dramatic respiratory emergencies typical in childhood which needs not only the appropriate endoscopic equipment suitable for the age, but also great experience in the management of the possible related complications. Massive hemoptysis is then discussed in order to help to choose the right endoscope and to clarify the steps requested to face this dramatic event. Lastly, iatrogenic tracheal injuries are described, in spite of their low occurrence. The correct endoscopic assessment of the lesions enables to select the proper multidisciplinary therapeutic approach together with surgeons and anesthetists. Due to their peculiarities, emergencies do not allow classic training so it is difficult to estimate the procedure volume necessary to achieve an adequate endoscopic experience. We think, in this field, it is advisable to refer to numbers proposed for elections endoscopic procedures. For these reasons, we consider desirable the use of simulators and clinic case discussions during interventional pulmonologist's training.

(Cite this article as: Simonassi CF, Majori M, Covesnon MG, Brianti A, Lazzari Agli L, Meoni E, et al. Competence in pulmonary endoscopy emergencies. Panminerva Med 2019;61:386-400. DOI: 10.23736/S0031-0808.18.03543-7)

KEY WORDS: Endoscopic procedures - Foreign bodies - Inhalation - Hemoptysis.

$\mathrm{T}$ here are many clinical entities representing a pulmonary endoscopic emergency, but two are the main pathophysiology mechanisms: obstruction of the central airway and the lost of anatomical barriers of the respiratory tract in the tracheo-bronchial lesions by trauma or by iatrogenic complications.

The main causes of the airway obstruction are determined by foreign body inhalation, massive hemoptysis, organic stenosis and atelectasis by secretion (after surgery or in chronic neuromuscular disease).

In this review we will discuss about foreign body inhalation, massive hemoptysis and iatrogenic tracheal injury.

Although in the late adulthood incidental inhalation of food may occur as the result of dysphagia associated to neurologic disease, a significant proportion of cases of tra- cheobronchial foreign body aspiration is observed during childhood. This kind of respiratory emergency is described in the section dedicated to pediatric emergency.

Massive hemoptysis is described in the endoscopic emergencies in adult people because it is rare in youth.

Lastly we will discuss iatrogenic tracheal injury (ITI), a rare condition which can complicate tracheal intubation.

\section{Inhaled foreign bodies in childhood}

Pediatric airway emergencies which require urgent bronchoscopic management include difficult airway (particularly in critically ill children and/or with craniofacial anomalies and polymalformative syndromes) ${ }^{1}$ and inhalation of foreign bodies (FBs). Massive hemoptysis is much 

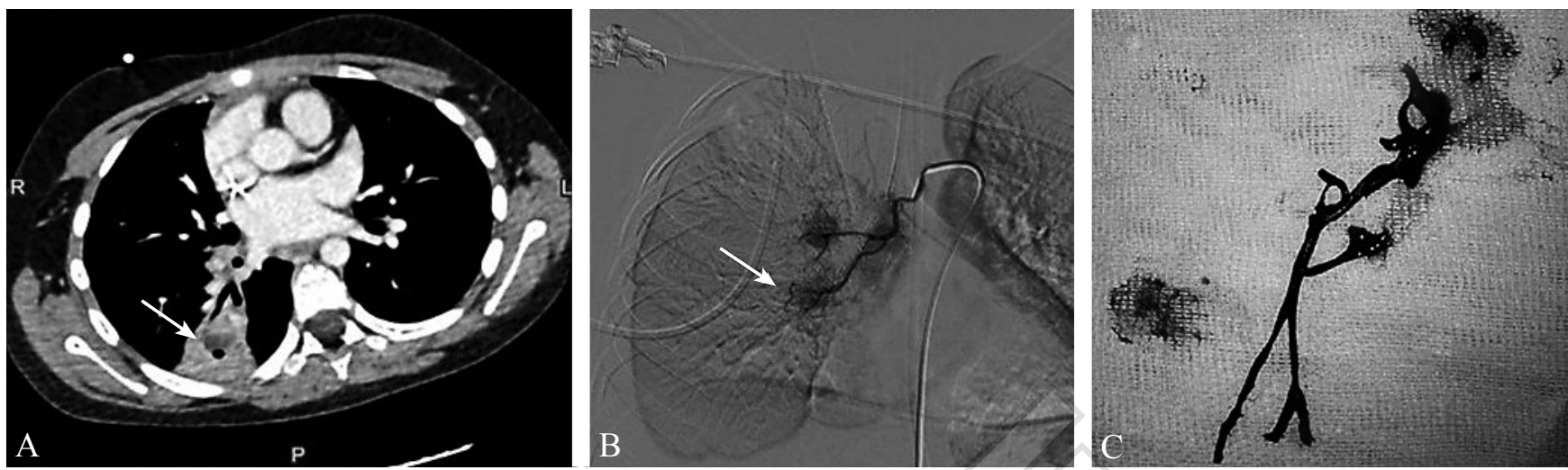

Figure 1.-A 7-year-old girl with life-threatening airway obstruction due to massive hemoptysis during chemotherapy for acute lymphoid leukemia complicated by pulmonary abscess (A, arrow). Hypertrophied right bronchial vessels supplying a marked hypervascular bed in the right lower lobe were angiographically assessed ( $\mathrm{B}$, arrow) and embolized following which hemoptysis ceased. A giant blood clot in the trachea, extending into and partially obstructing the right and left main bronchi was removed en-bloc (C) with using rigid bronchoscope.
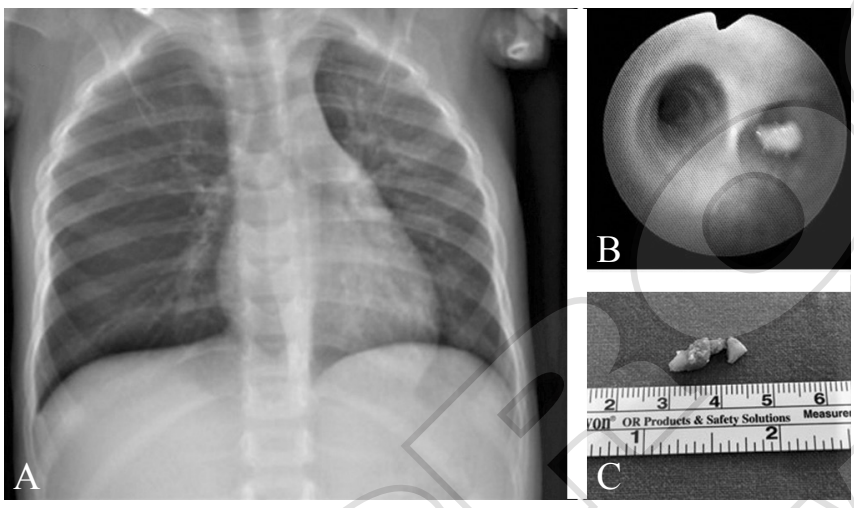

Figure 2.-Chest X-ray showing right-sided hyperinflation (A) due to peanut fragments completely occluding the right bronchial system (B: endoscopic view; C: FBs after extraction).

less common than in adults, although especially in immunosuppressed children with opportunistic infections may occur (Figure 1).

Knowledge of the disease

The inhalation of foreign body is a significant cause of morbidity in children: the complication rate is of $16 \%$ with $4.4 \%$ cardiorespiratory arrests and $6.2 \%$ deaths. $^{2}$

\section{Causes in different ages}

Approximately $80 \%$ of events occur in children aged $<3$ with the peak incidence between one and two years of age; the reasons for this are their habit to explore the surroundings with the mouth and to be active and playing while

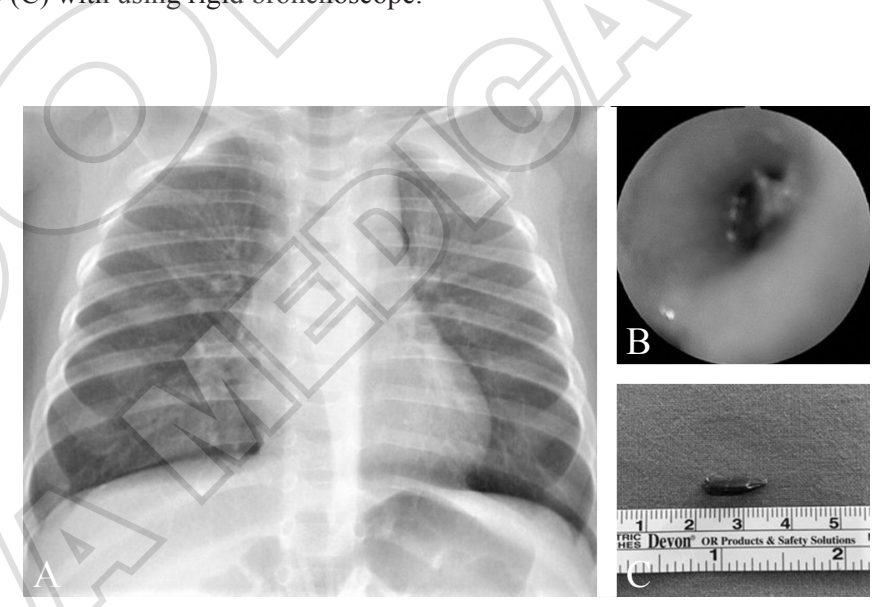

Figure 3.-Normal chest X-ray (A) despite a sunflower seed completely occluding the left main bronchus (B: endoscopic view; C: FB after extraction).

eating, their lack of molar teeth with poorer chewing, and their immature laryngeal reflexes. ${ }^{3}$

Organic materials, such as nuts (Figure 2) and seeds (Figure 3 ) are the most frequently inhaled FB $(67 \%$ of cases). ${ }^{4}$ Organic FBs can expand from bronchial secretions and worsen obstruction; moreover, materials with a high oil content (such peanuts) can also cause mucosal inflammation and accumulation of bulky granulation tissue leading to complication such as tracheobronchial mucosal bleeding during the extraction procedure.

The most common anatomic location for a FB is the mainstem bronchi $(80-90 \%)$, followed by the trachea (3$12 \%)$, and the larynx (2-12\%). The most frequent location in the right bronchial tree ( $60 \%$ of cases) is due to its close proximity to the vocal cords, more vertical disposition and 
to the position of the carina to the left of the midtrachea. ${ }^{5-7}$ The position of the carina to the right of the midtrachea could account for the prevalence of inhaled FBs in the left bronchial tree observed in some reports.

\section{Clinical history and physical exam elements}

The clinical presentation of FB inhalation depends on the size and localization of the FB. Laryngeal FBs can cause cyanosis and hypoxia with death from suffocation if the obstruction is complete. Tracheal and bronchial FBs can present as cough and/or dyspnea and/or cyanosis with or without vomiting (the so called "penetration syndrome"). However, these symptoms can subside spontaneously and quickly even when the FB remains; in this case, if the choking events is not witnessed or unrecalled, the child is at risk for complications (proportional to the delay of FB removal) as pneumonia, hemoptysis and bronchiectasis. So, when respiratory symptoms develop in an otherwise healthy child, one should always ask whether the child may have been inhaled something. The most important factor in diagnosis is considering the possibility!

\section{Radiographic interpretation}

The key to reach a definitive diagnosis lies in a detailed history that identifies risk factors, an examination that elicits important signs, and supportive chest X-ray findings. However, the inhalation may not have had witnesses, most inhaled FBs in children are radiolucent (up to $90 \%$ of cases) and physical examination may be normal (up to $56 \%$ of cases). ${ }^{2}$

When present, unilaterally decreased breath sounds and lung hyperinflation (Figure 1) are strong indicators of inhalation; in case of normal chest X-ray, a chest fluoroscopy (Figure 4) may be helpful to demonstrate the air trapping distal to the FB. Mediastinal contralateral shift can also be found.

Negative clinical and/or radiography should not exclude the diagnosis in patients with a strong history (sensitivity of 76 to $92 \%$ ).

Once FB inhalation is suspected, rapid confirmation is required. Even in stable patients, the potential for a FB to dislodge and possibly re-impact in the non-compromised lung should be considered and the removal delay should not exceed 12 to 24 hours. ${ }^{8}$

\section{Knowledge of instruments}

In children, different models of bronchoscopes are used depending on the age of the child. Currently available pediatric flexible bronchoscopes range in outer diameter sizes from 2.2 to $4.9 \mathrm{~mm}$. with a working channel range from 1.2 to $2.2 \mathrm{~mm}$. The $2.2 \mathrm{~mm}$ bronchoscope does not have a working channel and is therefore limited in its utility. The $4.9 \mathrm{~mm}$ scope can be used in children either over the age of 7 or weighing more than $20 \mathrm{~kg} .{ }^{9}, 10$ Pediatric rigid bronchoscopes are shorter than those used in adults and measure between 30 and $16 \mathrm{~cm}$ long with an internal diameter between $3.2 \mathrm{~mm}$ and $7 \mathrm{~mm}$.

In children, flexible bronchoscopy is used mainly for diagnosis and as an aid to intubation in the child with a difficult airway. Rigid bronchoscopy is the instrument of choice for diagnosis of glottic and subglottic pathology (cleft, high fistula), foreign body (FB) extraction, stent placement and laser surgery (strong recommendation; high-quality evidence). Pediatric rigid bronchoscopy is not performed in most centers of interventional pulmonology and no evidence-based method has been established to assess this competency. Therefore learning this technique usually requires training in a reference center.

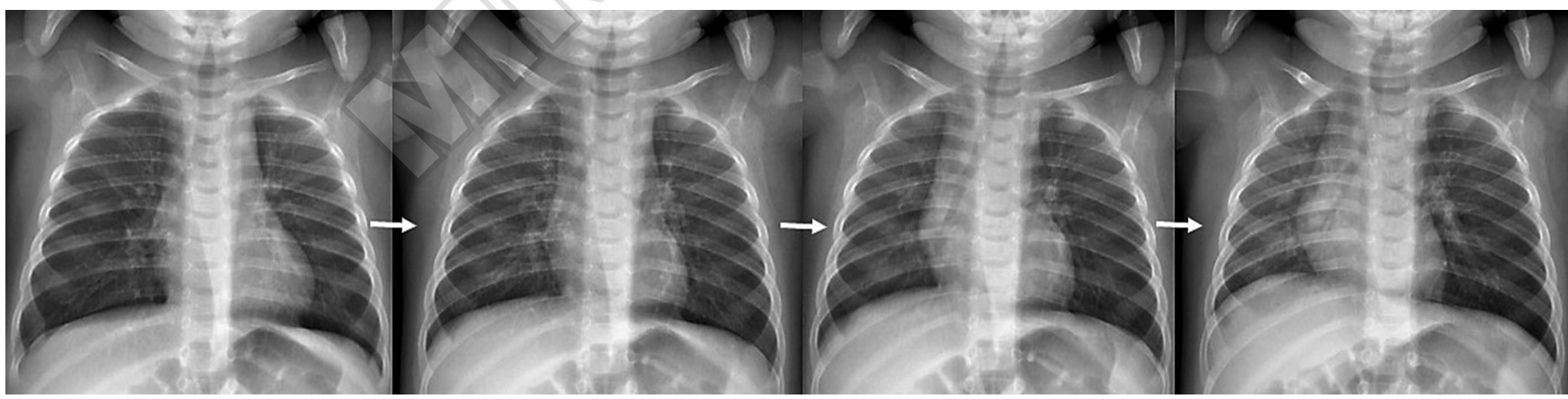

Figure 4.-X-ray fluoroscopy showing progressive left-lung hyperinflation with mediastinal shift due to the sunflower seed completely occluding the left main bronchus (see Figure 3). 
While flexible bronchoscopy can be done under sedation, rigid bronchoscopy always requires the use of general anesthesia. Type of anesthesia is an issue of controversy. There is a wide consensus on using sevoflurane or halothane as inhalational agents for induction of anesthesia and spontaneous breathing as a maintenance technique of choice. A strong coordination among interventional pulmonologist and anesthesiologist is essential to ensure that adequate oxygenation is maintained via the shared airway. Patients should be monitored with continuous pulseoximetry, cardiac and blood pressure monitoring. Supplemental oxygen and suction should be available at the bedside to be used as needed. Resuscitation equipment should be readily available in case of emergency.

Skills

The different anatomy and physiology of the pediatric airway compared to adult pose an entirely different set of challenges to interventional pulmonologist.

Mostly under three years of age, children have a:

- larger tongue relative to size of the oral cavity;

- higher and more anteriore larynx;

- glottic aperture partially covered by a floppy epiglottis and angle more acutely with respect to the trachea;

- reduced airways diameter that make that even a small reduction in their size can cause a significant increase in airway resistance;

- marked vagal response with tendency to laryngospasm and bradycardia, a higher rate of oxygen consumption and reduced functional residual capacity leading to tendency to hypoxia. ${ }^{11}$

The operator needs the following skills:

- understanding indications and contraindications;

- pre-bronchoscopy clinical and radiographic evaluation;

- potential difficulties in intubating the patient;

- choosing the appropriate procedure for the and the correct instrument (Flexible bronchoscope FB vs. Rigid bronchoscope RB);

- obtaining appropriate informed consent and evaluation of possible alternative procedures;

- patient safety;

- correct monitoring and ability to respond to abnormalities and achieve infection control;

- management of anaesthesia and sedation procedure in children (in collaboration with anaesthesiologists);

- ability to use of the basic supplies for paediatric bronchoscopy: slip-tip syringes, specimen traps, gauze sponges, bite blocks, appropriately sized bronchoscopes;
- knowledge of complications and their management;

- basic knowledge of instruments cleaning and disinfection;

- ability to introduce and use a rigid bronchoscope and related procedures (foreign body removal, stent, laser, etc.).

\section{Procedural steps}

Flexible bronchoscopy is the gold standard for definitive diagnosis of foreign body. Because of complications that can result from longstanding retained FBs, if there is any doubt about a possible inhalation, it is better to perform a bronchoscopy to conclude that there is not a FB rather than risk to leave a FB in the tracheobronchial tree. ${ }^{7,8}$

Although potential advantages of using flexible bronchoscopy for FBs extraction (i.e. not required general anesthesia and ability to reach more peripheral airways) have been reported, the instrument of choice to remove inhaled FBs in children is the rigid bronchoscope. ${ }^{12,} 13$ The use of flexible bronchoscope raises the risk for dislodging the FB and further compromising the ventilation; conversely, the rigid bronchoscope allows:

- control of the airway: the patient can be ventilated through the bronchoscope and - compared to endotracheal tube - with rigid bronchoscope the trachea is entered under continuous vision with possible identification of a subglottic or high tracheal FBs thus reducing the risk of sudden obstruction from dislodging it; 14

- use of strong forceps to securely grasp the FB: accidental loss of the FB or its fragments into previously healthy mainstem bronchus during the extraction procedure may be lethal if the originally involved lung is atelectatic or the originally involved bronchus remains obstructed by inflammation or residual FBs;

- use of large suction probes to manage the mucosal bleeding that can occur in the case of FB embedded in granulation tissue.

For smooth and rounded FBs the smooth forceps (foreign body forceps) should be preferred to the alligator forceps. The forceps' cups should be opened maximally to grip the largest volume of the FB without pushing the FB downwards. In case of technical challenges to grasp with forceps because of the shape of the inhaled FB endoscopic tipless baskets or balloon catheters can be used.

When the FB is larger than the working lumen of the rigid bronchoscope, both forceps and FB are pulled up, a few millimeters distal to the tip of the bronchoscope, and then the instruments and the FB are withdrawn together from the trachea. In case of a sharp FB stuck in the tra- 
cheobronchial wall, to prevent the risk of wall laceration and/or massive hemoptysis, it should be pushed down to dislodge the tip; then, the tip should be placed into the RB beveled end before extraction attempt.

During the last step of extraction procedure, the FB can be lost accidentally, either because there was some inappropriate coaxial movement between the bronchoscope and the forceps or because it is blocked in the narrow glottic area. If this occurs, the interventional pulmonologist should first carefully inspect the oral cavity and the larynx prior to re-entered the trachea with the rigid bronchoscope.

Once the FB is removed, the trachea should be reintubated with the rigid bronchoscope to re-examine the airways to rule out the presence of another FB or residual fragments. This is usually done by inserting a flexible bronchoscope through the rigid bronchoscope. The presence of small fragments in more peripheral airways (where they can also dislocate as a result of vigorous grasping of friable FB) may be challenging for the interventional pulmonologist, mostly when they reach the limit of flexible bronchoscopy accessibility. In this case, the FB fragments can be sucked through a suction probe or dislodged with aid of topical application of adrenalin or saline washes. When all these measures fail, the procedure should be stopped and rescheduled after antibiotics and steroid therapy. ${ }^{14}$ Surgical resection of the segment or partial lobe is required for only $1.2 \%$ of the patients.

The timing of bronchoscopy and the therapeutic strategy depends on the clinical picture. ${ }^{8}$ In case of asphyxia or severe dyspnea the child should immediately undergo a rigid bronchoscopy; in all other cases we suggest that flexible bronchoscopy be done first to:

- confirm the diagnosis;

- define the nature and localization of the inhaled FB;

- choose the appropriate instruments for the extraction procedure.

The interventional pulmonologist must be aware that, even during the diagnostic procedure an accidental dislodgement of the FB can happen; thus, the interventional pulmonologist performing the diagnostic bronchoscopy must be able to promptly shift from a flexible bronchoscopy into a rigid one and also diagnostic flexible bronchoscopy should be performed in an operating room. Likewise, in case of extraction attempt of an inhaled FB with the flexible bronchoscope, the immediate availability of a rigid instrument is strictly recommended to guarantee safety of the procedure if the flexible bronchoscopy fails. ${ }^{12}$
Theoretical and practical training: resources for "Core Competences"

- Video libraries;

- training on plastic manikins of infants and children;

- simulation on self-made phantoms, on animal models such as pig lung, either frozen or preserved under plastic lamination;

- virtual simulation trainers used for adult bronchoscopy, which can be adapted to training for paediatric age patients;

- theoretical and practical courses: i.e. ERS Skills Based Course $(25 \%$ of the course should address theoretical aspects of flexible bronchoscopy in children; $25 \%$ should be taken up by discussion of clinical cases; $50 \%$ of course should be practical);

- training on patient under supervision until trainee has achieved quantitative and qualitative competence;

- attend procedures in endoscopy room at high volume centres of excellence.

Qualitative and quantitative assessment

Quantitative assessment:

Number of procedures (once trainee has achieved competency in adults):50

Qualitative Assessment:

- MCQ;

- case-based questionnaires on appropriate decisions;

- DOPS with flexible and rigid bronchoscope on manikin and patient.

Outcome measures: Indication, diagnostic yield, complications, quality of documentation, and overall cost-effectiveness.

\section{Emergencies in adult patients}

The main indications are hemoptysis, tracheobronchial stenosis and injuries, foreign bodies.

Bronchoscopy can be used to investigate hemoptysis. CT (computer tomography) scans have changed diagnostic procedure protocols and should always be considered prior to bronchoscopy. Consider bronchoscopy after a normal CT if the patient is high risk for lung carcinoma or if the hemoptysis continues.

Training should therefore be focused on the management of bleeding, tracheobronchial stenoses and injuries.

The tools for practical training include low and highfidelity simulation, although non-technical skills and team simulation are especially recommended. 
Trainers must have completed training described in sections on: "Flexible bronchoscopy and basic biopsy technique" and "Operative bronchoscopy procedures."

\section{Hemoptysis}

Knowledge of the disease

Chest physician is often called to evaluate hemoptysis. ${ }^{15}$

In the majority of the cases, expectoration of blood from the lower respiratory tract is mild to moderate and the diagnostic and therapeutic process can be conducted step by step.

Although massive hemoptysis is less frequent, it can rapidly compromise airways, therefore it should be viewed as a medical emergency requiring prompt intervention and a safe use of the dedicated instrumentation.

Massive hemoptysis occurs in between 5\% and 15\% of patients presenting with lower airways bleeding. 16, 17 There is no clear consensus on its precise definition, as shown in Table I.18-21

No uniform cut-off value is agreed in the literature so, massive hemoptysis definition relies on the main clinical consequences as hospitalization, anemia requiring transfusion, hemodynamically instability and hypoxemia requiring intubation, death. ${ }^{22-25}$

\section{Causes}

Causes of known hemoptysis: Bronchial circulation is responsible for $90 \%$ of massive hemoptysis. Systemic circulation, as aorta or non-bronchial arteries (subclavian artery and ancillary as thyrocervical trunk, internal thoracic artery; intercostal arteries, the upper and inferior phrenic arteries, coronary arteries) accounts for about $5 \%$ of bleeding, whereas pulmonary circulation is reported to be the source of bleeding up to $5 \%$ of cases. ${ }^{26,27}$

Several diseases may cause hemoptysis with different incidence related to the concerned geographic area. In western countries massive hemoptysis associated to lung cancer is reported to cause up to $35 \%$ of hospital admissions. Bronchogenic carcinoma is the most common cause, followed by bronchial carcinoid and metastatic lesions to the lung.

TABLE I.-Massive hemoptysis: quantitative definition.

\begin{tabular}{|c|c|}
\hline Amirana ${ }^{18}$ (1968) & $100 \mathrm{~mL} / 24 \mathrm{~h}$ \\
\hline Crocco ${ }^{19}$ (1968) & $600 \mathrm{~mL} / 24 \mathrm{~h}$ \\
\hline Corey ${ }^{20}$ (1987) & $1000 \mathrm{~mL} / 24 \mathrm{~h}$ \\
\hline Hirshberg ${ }^{21}$ (1997) & $500 \mathrm{~mL} / 24 \mathrm{~h}$ \\
\hline
\end{tabular}

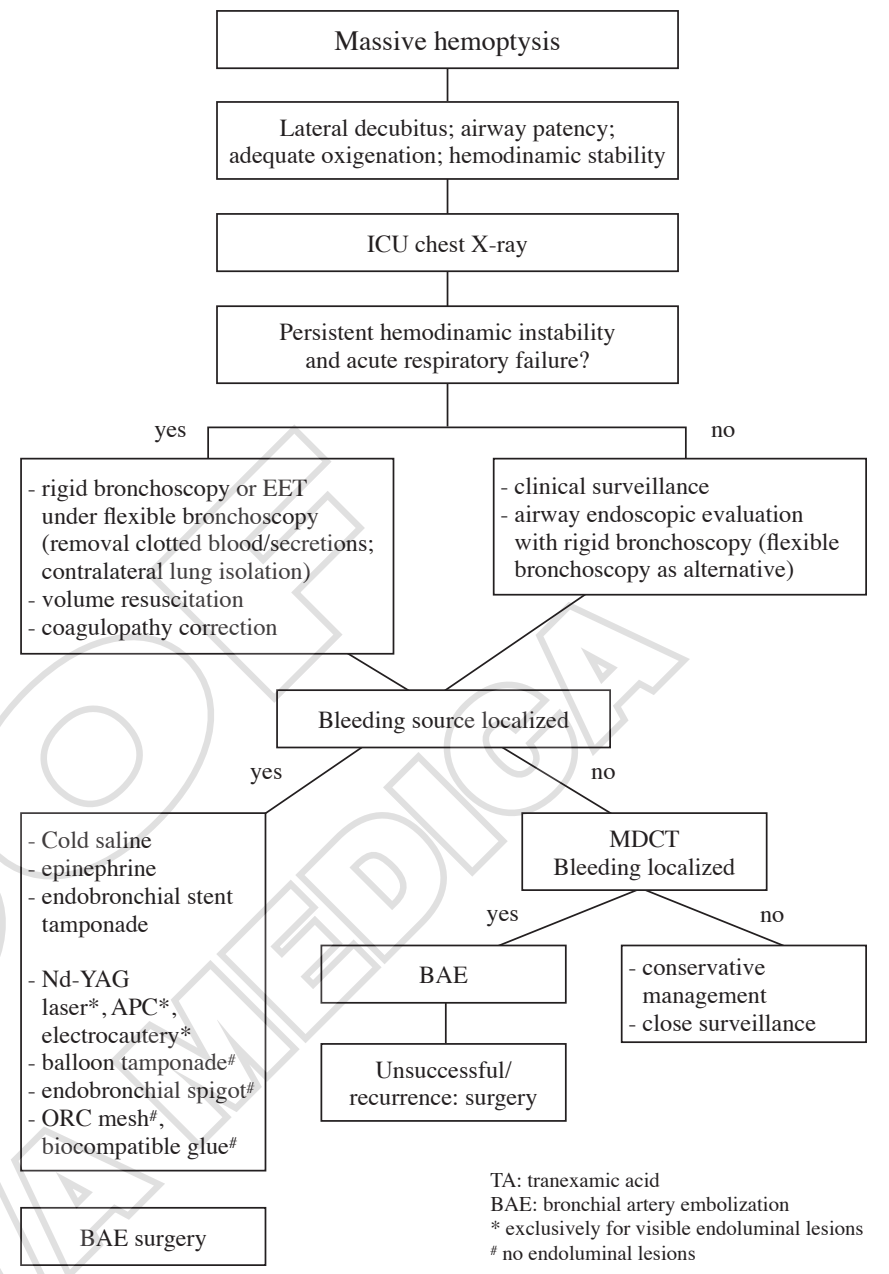

Figure 5.-Management of massive hemoptysis.

Bronchiectasis and tuberculosis are common causes of airway bleeding. ${ }^{28}$ In the literature cryptogenic hemoptysis may account up to $50 \%$ of cases. 15

In developing countries, tuberculosis and infections remain a prominent cause of hemoptysis. Angio-invasive fungal infection and necrotizing pneumonia (invasive or chronic necrotizing pulmonary aspergillosis) may lead to massive hemoptysis especially in immunocompromised hosts. ${ }^{29}$

Other causes are vasculitis, coagulopathy, iatrogenic lesions, trauma, vascular malformation (Table II).

\section{Treatment flowchart for massive hemoptysis}

Hemoptysis is often an alarming symptom. Major hemorrhage may dramatically lead to death depending on asphyxiation rather than on the induced hemodynamic in- 
TABLE II.-Causes of massive hemoptysis.

\begin{tabular}{ll}
\hline Neoplastic & - Bronchogenic carcinoma \\
& - Airway neoplasms (carcinoid, adenoid cystic carcinoma) \\
& - Lung metastases \\
Infectious & - Bronchiectasis \\
& - Bronchitis \\
& - Abscess \\
& - Fungal infectious (especially mycetoma) \\
Hematologic & - Coagulopathy (congenital; acquired mostly iatrogenic) \\
disorders & - Thrombocytopenia or platelet disfunction \\
Vasculitis & - ANCA associated vasculitis \\
& - Goodpasture syndrome \\
& - Systemic lupus erythematosus pneumonitis \\
Vascular & Bronchial and pulmonary artery aneurysm (Rasmussen \\
& - Aneurysm) \\
& - Pulmonary embolism \\
Miscellanea & - Cryptogenic \\
& - Lung/airway trauma accidental or iatrogenic (biopsy and \\
& intubation induced) \\
& - Endometriosis \\
& - Lymphangioleiomiomatosis \\
& - Mitral stenosis
\end{tabular}

stability. Mortality rate is from 9 to $38 \%$ of cases, even though in recent years we noticed an improvement related to technological advances in interventional radiology (Figure 5). ${ }^{21,30}$

Diagnostic approach consists in an accurate and prompt clinical history and a deep physical examination: it is not easy since this symptom causes anxiety and patients can overestimate the bleeding.

It is essential to determine if the hemorrhage is com- ing from the airways and not from the oral cavity or gastrointestinal tract (pseudo hemoptysis). Reported cough is helpful because usually shows hemoptysis. Other parameters as secretions $\mathrm{pH}$ or food in the expectorate, are not easily evaluated in the context of massive hemoptysis and scarcely useful in the diagnostic approach. Thoracic imaging (computed tomography and angiography) has a pivotal role and may be delayed only in few critical patients.

CT scan should be performed prior to bronchoscopy especially in patients with increased cancer risk (male, age $>40$ years, smoking history). It can detect lung neoplasms even when chest radiography is normal and is as efficient as bronchoscopy in detecting the site of bleeding in major bleeding. 31, 32 Nevertheless, bronchoscopy has an undeniable role in the management of hemoptysis because it can obtain samples for microbiology and pathology examination to come up with the etiologic diagnosis.

While the role of bronchoscopy is still debated in young people without cancer risk at their first episode of bleeding from the respiratory tract and with normal chest X-ray, there is no doubt about its need in case of massive hemoptysis (Figure 6) as a diagnostic and therapeutic tool. 33

Bronchial artery embolization (BAE) is the most effective treatment for refractory and massive hemoptysis, it provides immediate control of bleeding in $66-96 \%$ of patients.

Pathological signs of bleeding vessels are increased diameter $(>3 \mathrm{~cm})$, tortuosity, neovascular /hypervascularity, extravasation of contrast medium, presence of aneurysm changes.

It could be performed by arterial access: 1) femoral artery more common, lower morbidity; 2 ) brachial artery.

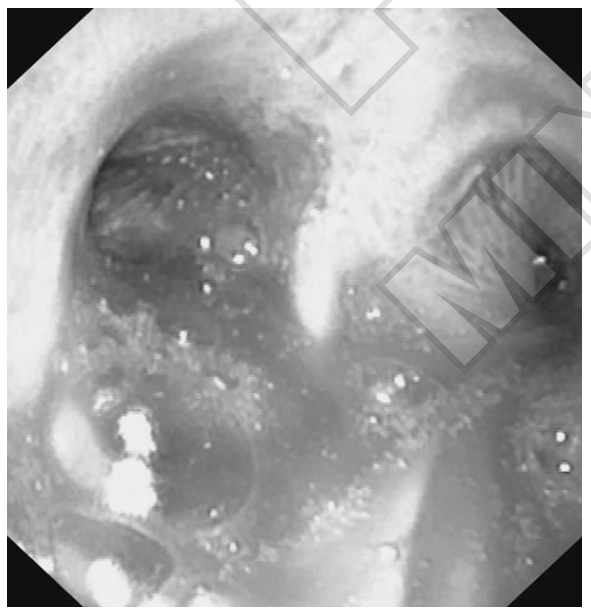

Figure 6.-Massive hemorrhage from the left main bronchus.

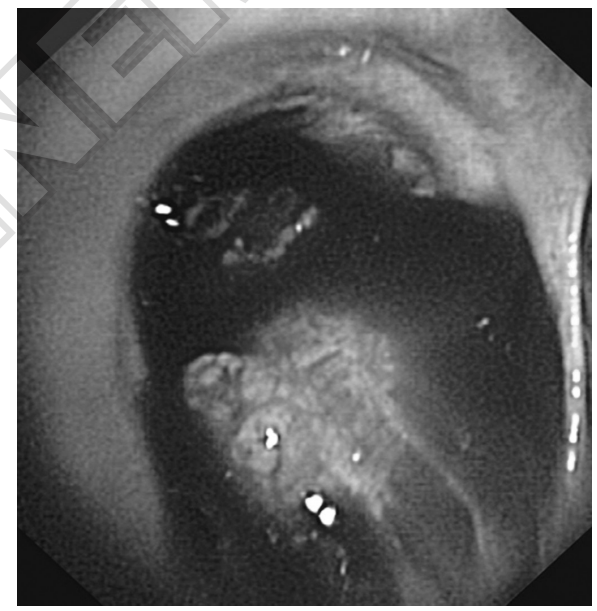

Figure 7.--Vegetative hemorrhagic lesion of the left main bronchus.

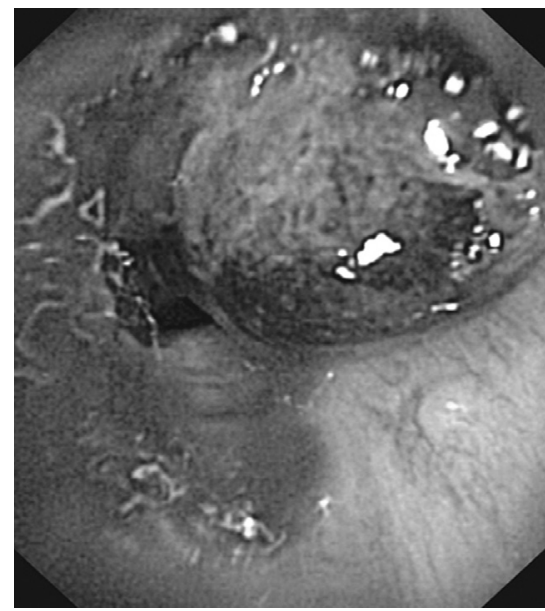

Figure 8.-Vegetation of the right main bronchus with high hemorrhagic risk. 
Artery cannulation is obtained with a $5 \mathrm{Fr}$ angiographic pigtail catheter (Cook Medical ${ }^{\mathrm{TM}}$, Limerick, Ireland) or superselective cannulation with a $2.7 \mathrm{fr}$ (Progreat ${ }^{\mathrm{TM}}$, Terumo, Japan) or 3 Fr microcatheter (Cook Medical ${ }^{\mathrm{TM}}$ ).

Artheriography should be performed with low osmolar or iso-osmolar non-ionic contrast material agents used for embolization Gelfoam and polyvinyl alcohol (PVA) particles. Microsphere. The choice of the agent to use depends on operator experience and availability of the embolic material.

Thoracic pain, embolization of the anterior spinal artery leading to anterior spinal cord syndrome might be complications.

\section{Knowledge of instruments}

Rigid and flexible bronchoscopes could be used in case of massive haemoptysis.

The first one offers these advantages: 1) best control of airway patency through the possibility of adequate suction for evacuating bloods and clots 2) allows concurrent ventilation of the patient 3) enables the use of different tools to remove airway obstruction (Figure 7).

Flexible bronchoscope is more manageable, less traumatic and provides easier access to the upper lobes and peripheral bronchi.

From the previous description, it seems that the first line of evaluation and treatment of massive haemoptysis should rely on rigid bronchoscopy, ${ }^{34}$ but intubation of patients with the rigid scope is difficult under critical conditions because it is less manageable and at risk to be traumatic so, rigid bronchoscope should be used in well trained centers. Further, planning the exam takes time, which is a disadvantage when urgent endoscopy is needed. Therefore, we suggest as good practice the choice of the instrument which best fits the physician's skills, keeping the rigid bronchoscope as the gold standard in situations at risk for major bleeding (Figure 8).

We recommend not to perform biopsy in particular endoscopic situations. Bronchial Dieulafoy's lesion represents a paradigmatic example. It is a small non-throbbing formation covered in white mucous membrane originated from an arteriolar dilation of bronchial or (less frequently) pulmonary circulation located in the underlying tissue. When biopsied, it could lead to massive bleeding, in some cases even fatal. When a Dieulafoy's disease is suspected, biopsy shouldn't be performed but rather ecographic evaluation of submucosal blood vascular abnormalities is needed, leaving embolization to a late angiographic study. 35

When performing bronchoscopy, we must remember the risk of bleeding related to severe coagulopathies (platelet count lower than 50000 per $\mu 1$ for endobronchial biopsy and platelet count lower than 30000 per $\mu 1$ for TBLB; INR greater than 1, 4 or t.Quick $<60 \%$ ) or patients receiving therapeutic anticoagulation or drugs inhibiting platelet function. ${ }^{36}$

These drugs have been associated to an increase rate of spontaneous bleeding and should therefore be withheld before performing invasive bronchoscopic interventions according to guidelines, considering and balancing the risk of thrombotic complications. ${ }^{37-39}$

Skills

1. Selection and interpretation of appropriate imaging to assist in the diagnosis of hemoptysis

Chest radiography is inexpensive, ubiquitous, the initial imaging study.

Limitations: it provides bleeding localization in $46 \%$ of cases; normal chest radiography in $25 \%$ of patients with malignancy.

Multidetector CT (MDCT) scan is performed in the setting of gross and recurrent hemoptysis in patients at risk for lung cancer. MDCT scan with contrast is the gold standard.

MDCT angiography is highly accurate in characterizing thoracic bronchial ectopic, non-bronchial and pulmonary arteries.

The time required to obtain images, renal dysfunction and the supine position of the patient may be some limitations.

2. Ability to use the technique for airway control and oxygenation/ventilation

The management of massive hemoptysis consists in three steps: 1) maintaining airway patency and patient's clinical stability; 2) localizing the bleeding source 3) specific therapy delivery. 40

First of all, the patient should be placed in a lateral decubitus position on the bleeding side in order to avoid aspiration of blood in the controlateral lung.

Hemodynamic stability is ensured by delivering supplemental oxygen, fluid and correction of any coagulopathy that may contribute to the bleeding.

\section{Ability to selectively intubate the patient with flexible bron- choscopy}

Bronchoscopy performed by flexible fiberoptic bronchoscopy (FOB) is used to localize the bleeding source (diagnostic aim) and to remove clotted blood and secretions (therapeutic aim). 
In massive hemoptysis associated to persistent hemodynamic and respiratory instability, it is pivotal to safeguard airways patency by a large-bore endotracheal tube (ETT) under fibreoptic guide. Afterward we can perform a complete exploration of the bronchial tree through the ETT. Alternatively, a skilled physician could attempt urgent intubation with rigid bronchoscope.

Given the critical clinical conditions and the needs to maintain airway patency, Intensive Care Unit (ICU) is the ideal setting for patients with massive hemoptysis.

\section{Ability to administer correctly drugs such as adrenaline, ornipressin and tranexamic acid}

Treatment of the bleeding source is the next step. Bronchoscopic therapy can ensure definitive control of bleeding, very important especially in patients not fitting for surgery or in patients allergic to contrast. Otherwise, this approach is often a temporary measure, as bridging treatment to bronchial artery embolization (BAE) or surgery.

Efficient treatment of bleeding airways are: 1) topical endobronchial application of pharmacological agents; 2) laser photocoagulation of the visible lesion; 3) mechanical therapy with physical occlusion of the bleeding airway segment.

In the " 80 s some authors reported the efficacy of repeated irrigations with iced saline solution at $4{ }^{\circ} \mathrm{C}$ in 50 $\mathrm{mL}$ aliquots on average $500 \mathrm{~mL}$ per patient with suction of liquids and clots. ${ }^{41}$

Since instillation of big amounts of liquid reduce airway patency, this approach is restricted to mild- moderate bleeding.

Some studies have reported local instillation of topical vasoconstrictive agents in the bleeding airway, as epinephrine and vasopressin analogues, terlipressin and ornipres$\sin$.

This approach is not useful for massive bleeding because the drug is diluted and washed away by blood. Further, epinephrine and terlipressin have been reported to cause significant cardiovascular effects namely tachyarrhythmia and acute hypertension related to high plasma level following endobronchial application. ${ }^{42-44}$

Topical use of tranexamic acid, an antifibrinolytic drug, has been successfully reported in the past in patients with cystic fibrosis. 45,46

Endoscopic instillation of fibrinogen-thrombin complex (FT) has shown good results. Described in several recent studies, the injection of FT showed a good prompt prothrombotic effect and immediate control of massive bleeding, so it seems to be a promising strategy in the early management of massive hemoptysis in patients unable to undergo surgery. 47,48

Some authors described the efficacy of substances with hemostatic properties useful in case of massive persistent bleeding. Valipour in the ' 80 s tested an absorbable water-insoluble derivate of cellulose (ORC mesh: oxidized regenerated cellulose). The mesh was placed selectively into the bleeding bronchus grasped with biopsy forceps through the working channel of a bronchoscope. With blood contact, this fabric became a brown plug which contributed to clot formation. The apposition of at least 4 to at most 10 layers of the fabric could stop the bleeding. This way of bleeding control was effective in $90 \%$ of cases but, obviously is not indicated when the source is in central airways. ${ }^{49}$

Some authors described the use of endobronchial biocompatible glues as N-butyl cyanoacrylate solidifies on contact with humidity. This material was injected into the bleeding airway through a catheter via a flexible fiberoptic bronchoscope to achieve sealing. This technique is not indicated in elderly patients with cardiopulmonary instability, in case of diffuse bleeding or when the sealed airway could exclude from ventilation a significative amount of the dependent lung. ${ }^{50,51}$

It is important to remember that in massive hemoptysis, blood expectorated dilutes the antifibrinolytic agents thus reducing their efficacy.

\section{Ability to perform a mechanical tamponade with balloon,} blocker tubes and rigid bronchoscopy

When the source of bleeding is a bronchoscopically visible lesion, an effective treatment option is the debulking of the lesion after devascularization by heat therapy (laser photocoagulation, argon plasma coagulation, electrocautery). Sometimes it can be a definitive treatment.

In life-threatening hemorrhage, mechanical therapy allows physical occlusion of the bleeding airway segment and prevents spillage of blood into the healthy lung segments.

Endobronchial blockers are the most frequently used devices to stop bleeding.

In the ' 70 s, some authors used to stop bronchial bleeding placing a Foley catheter through the rigid bronchoscope or an endotracheal tube. Others used to position a Fogarty balloon catheter introduced under visual control through the flexible bronchoscope and kept in place until surgery.

To overcome these limitations, Freitag developed bronchial blockers (BB) usually inserted into the working channel of an FOB and kept in place after its removal (Figure 9, 10). ${ }^{52}$ 


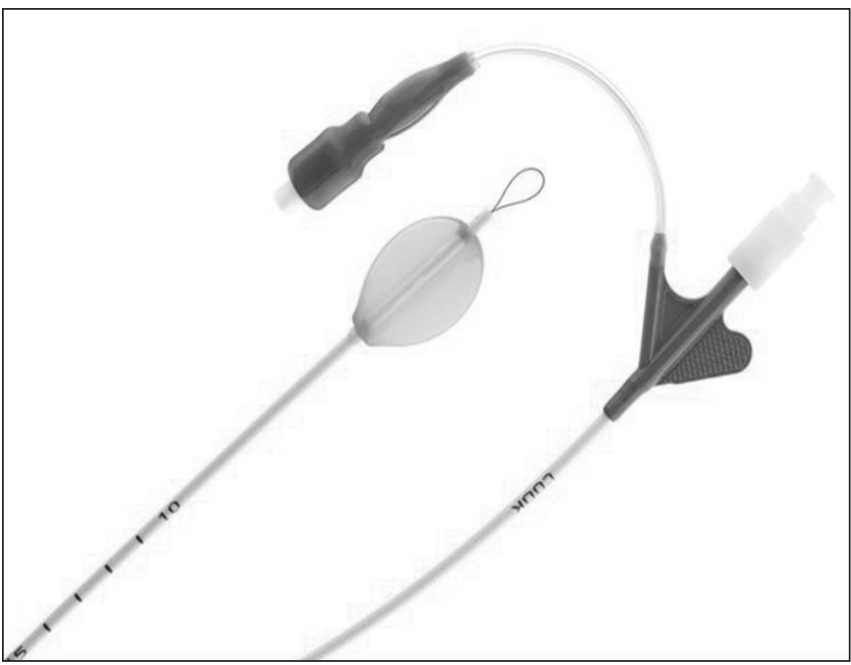

Figure 9.- Arndt bronchial blocker.

These devices allow therapeutic bronchoscopy treatment as distal secretion suction and instillation of vasoactive/antifibrinolytic agents. Their disadvantage is the difficult placement in the upper lobe bronchi and their dislocation induced by cough (Figure 11, 12).

Silicone spigots, originally used in the treatment of bronchopleural fistula, have proved to be effective for temporary control of bleeding from segmental bronchi, allowing patients to stabilize before BAE. 53

When all the above devices are unable to stop bleeding, isolation of the healthy lung is the last chance. It can be achieved by selective intubation with a double-lumen endotracheal tube (Carlens e Robershow); the main disadvantage is technical difficulty in properly positioning.

Univent bronchial blockers overcame this limitation, it has a channel embedded in its wall, its distal tip is curved

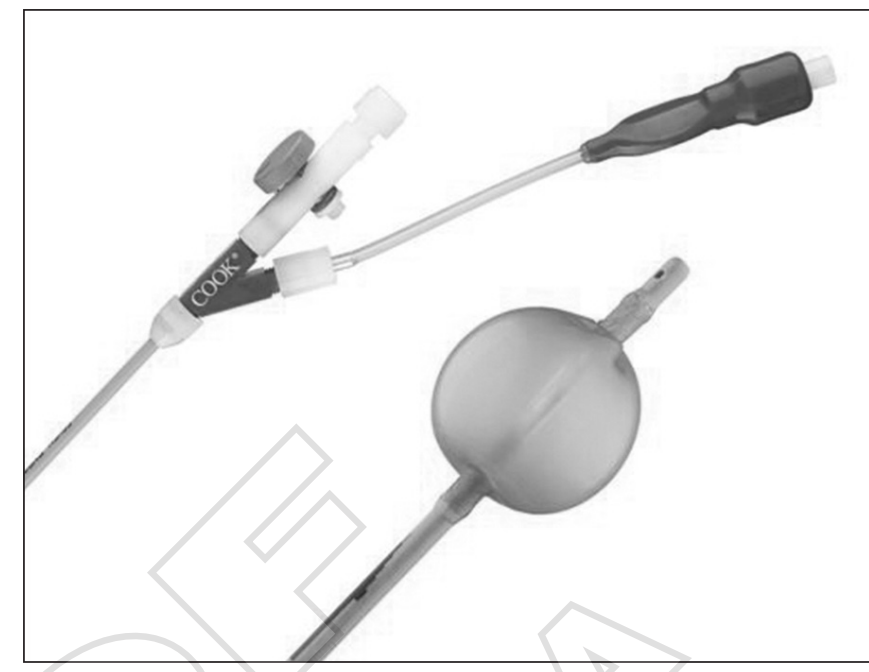

Figure 10.- Cohen bronchial blocker.

and rotation of the proximal end of the $\mathrm{BB}$ allows to guide its distal end into the desired mainstem (Figure 13).

\section{Procedural steps}

Significant unexpected bleeding:

- ensure adequate oxygenation and IV access for fluid resuscitation. Vital signs should be monitored regularly;

- retract the bronchoscope proximally to maintain vision; apply suction to remove free blood so as to preserve airway patency;

- consider lying patient onto the side of the bleeding. Do not suction to remove clot;

- consider the application of local vasoconstrictor therapy. Agents include 5-10 mL 1:10000 epinephrine or 5-10 $\mathrm{mL} 4{ }^{\circ} \mathrm{C}$ saline. Saline has the advantage that it may be administered repeatedly;

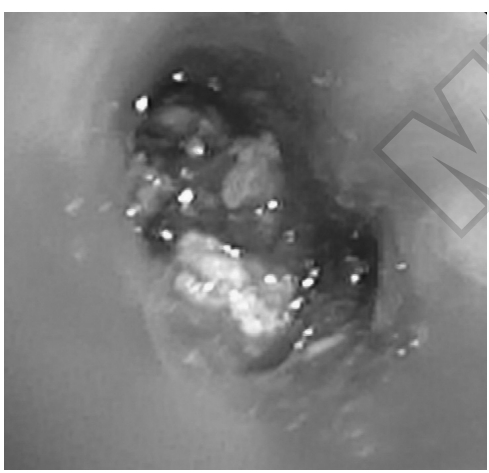

Figure 11.- - Hemorrhagic lesion of left upper lobar bronchus.

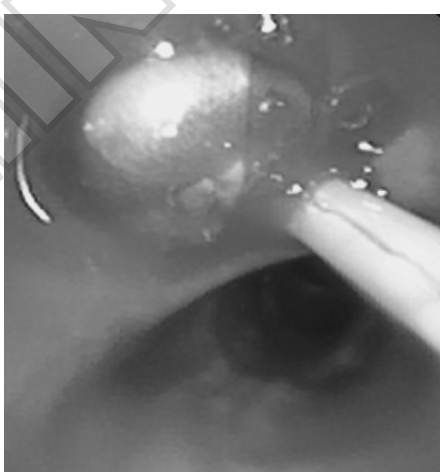

Figure 12.-Use of blocker for bleeding from left upper lobar bronchus.

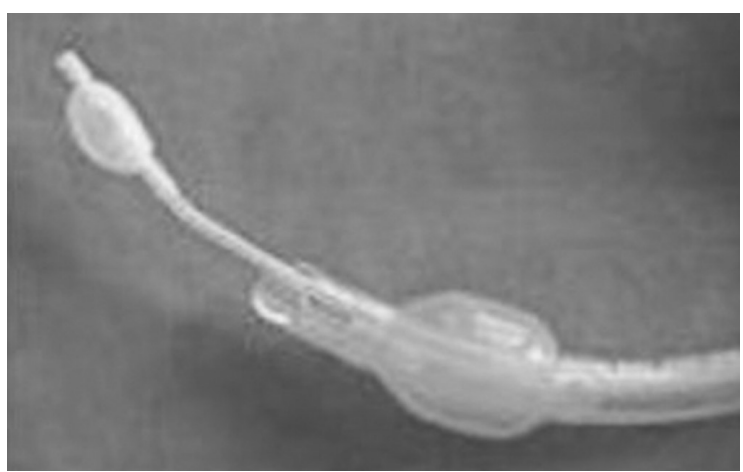

Figure 13.--Univent tracheal tube. 
- if bleeding continues, the bronchoscope should be wedged into the bleeding segmental bronchus, if possible, and held in place for 10-15 minutes;

- if this does not control the bleeding, a balloon catheter can be used to apply pressure and isolate the segment;

- check platelet count, PT and PTT, and recheck drug history;

- seek senior/expert assistance, and consider referral to critical care.

Bleeding after biopsy (CHEST live learning) includes five steps:

- step 1:1) wedge the bronchoscope; 2) maintain wedge position after biopsy (1-2 minutes); 3 ) avoid suctioning after biopsy;

- step 2:1) safety position (lateral decubitus); 2) turn the patient onto a safety position (bleeding side down) to protect the contralateral airway; 3 ) allow face to face contact with patient if operator working from the front or side of the patient; 4) allow blood and secretions to flow from the larynx and out of the corner of the mouth; 5) avoid collapse of the larynx and laryngeal obstruction by tongue or edematous upper airway; 6) oral pharynx easily suctioned;

- step 3: saline lavage, immediate administration of large aliquots of iced saline using a wedged or partially wedged bronchoscope and continuous or intermittent suction and gravity dependent clot formation, which will stop most bleeding;

- step 4: intubation with cuffed endotracheal tube. 1) If bleeding persists and airway is compromised, intubate the patient; 2) selective main stem intubation may be necessary;

- step 5: insert bronchial blockers (the bronchial blocker can be inserted inside or outside endotracheal tube), bronchial blockers placement into bleeding site may be considered.

\section{Theoretical and practical training}

During a medical emergency, every step and decision is crucial, so interventional pulmonologist have to attend extensive training to acquire technical expertise in endoscopic procedures.

In order not to improve endoscopist's expertise directly on patients, putting their life at risk, current training programs relies on the use of high-fidelity simulation tools. BRONCH Mentor Symbionics has a specific module for hemoptysis. Simulations provide trainees to develop their skills in clinical scenarios like those of hemoptysis, before acting in real life. The optimal educational approach con- sists in discussion of clinical cases with a supervisor so that students can strengthen the decision-making process.

Management of massive hemoptysis is complex and involves the use of rigid and flexible bronchoscope, the capability to endotracheal intubation by FOB and the use of BBs. For these reasons interventional pulmonologist has to develop competence on these techniques with proper training programs.

Non-technical skill scenarios permit to simulate experiential situations.

\section{Qualitative and quantitative assessment}

Several training programs tried to establish the number of endoscopic procedures needed to get a good competence level in standard situations, defining 20 exams with the rigid scope and 100 with flexible bronchoscopy. ${ }^{54}$ Qualitative and quantitative assessment of the competence could be monitored by multiple choices questionnaire, casebased discussion and direct observation of experts. $.5,56$

Given the scarce predictability of medical emergencies, it is not possible to quantify the volume thresholds for endoscopic procedures to demonstrate the competence required.

Outcome measures: Indication, diagnostic yield, complications, quality of documentation, and overall cost- effectiveness.

\section{Iatrogenic tracheal injuries}

Iatrogenic tracheal injury (ITI) is a complication of endotracheal intubation and an endoscopic emergency . It is a rare condition, which percentage is not easy to calculate. Some authors, on the basis of retrospective study, reported an incidence of a lesions on 20.000-75.000 intubations, and in the last decade we estimate an incidence of 0.05 $0.37 \% .{ }^{57}$

Risk factors are mechanic and physiologic factors (Table III): it is important to remember that emergency intubation is the most important risk factor.

ITI is a severe condition and it needed a prompt diagnosis and management to patient's survival. ${ }^{58}$

After intubation in case of initial signs of complication (subcutaneous emphysema, dyspnea, cough or hemoptysis) ITI should be suspected.

Usually it happens in the first hour after extubation within 12 hours. Only in patients who underwent thoracic surgery the diagnosis could be done immediately.

A thoracic computed tomography has to be performed to demonstrate: subcutaneous emphysema, pneumotorax, pneumomediastinum, or fluids in the mediastinal space. 
TABLE III.-Iatrogenic tracheal injury: risk factors.

\begin{tabular}{ll}
\hline \multicolumn{1}{c}{ Mechanical } & \multicolumn{1}{c}{ Anatomic } \\
\hline - Repeated attempts & - Congenital anomalies \\
- Inexperience & - Deviation trachea (goiter and \\
- Spindle & growths) \\
- Patient repositioning with cuffed & - Pre-existing strictures \\
tube & - Subtlety pars membranacea \\
- Inappropriate tube size & - COPD \\
& - Female sex \\
& - Age $>50$ \\
& - Height $<165 \mathrm{~cm}$ \\
\hline
\end{tabular}

Direct examination with bronchoscopy remains the gold standard for ITI.

Currently there is no unanimous consensus on the therapeutic management of ITI, ${ }^{58-62}$ based on variability on clinical presentation and anatomy of the tracheal injury.

Until the '90s, pneumologists preferred early surgical repair. The aims of this treatment were: avoiding air leaks with consequent impaired ventilation, preventing mediastinal infection and reducing stenosis complications.

Differently from what happens in blunt injury, further studies showed that in the ITI there are few air leaks, and that mediastinal infection and stenosis are rare.

The reason of these discrepancies may be the different mechanism responsible of the two types of lesion. ${ }^{63}$

Some authors suggest a conservative treatment and follow-up bronchoscopy (Figure 14, 15) in patient with respiratory autonomy, superficial injury or in which extubation could be perform in the first hours after signs presentation. ${ }^{64-69}$

Initially it was proposed only for superficial and small lesions $\left(<1 \mathrm{~cm}\right.$ length for Kaloud, $<4 \mathrm{~cm}$ for Jougou). ${ }^{70-71}$ Conti et al. in 30 consecutive patients (15 mechanical ventilation, 15 breathing) obtained a resolution of the rupture

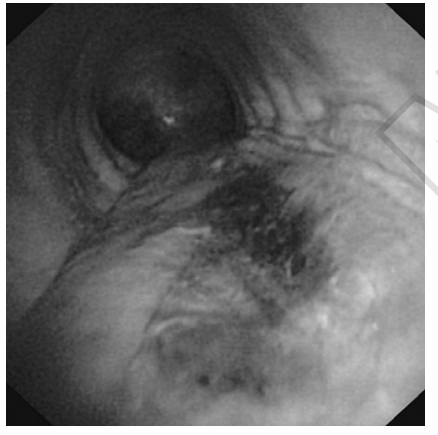

Figure 14.-Iatrogenic tracheal injury subjected to conservative treatment.

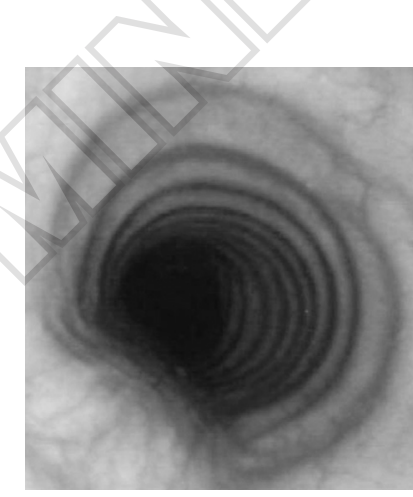

Figure 15.-Final result after conservative treatment. with a conservative management in all the patients no requiring mechanical ventilation and the $70 \%$ of those undergoing mechanical ventilation..$^{72}$

Authors agree that spontaneous breathing is fundamental when choosing treatment, since other parameters are not as important, thus a conservative management is recommended as a better choice..$^{73}$

If MV is needed, the implementation of conservative treatment presupposes the possibility of bridging the lesion, placing downstream the insufflated cuff of the tracheal tube. If this is not possible and NIV is not feasible, after assessing the risk, it is necessary to initiate the patient to surgical therapy. ${ }^{74}$

\section{Knowledge of instruments}

Direct examination with bronchoscopy allowing to confirm the clinical suspicion, to localize the lesion and assess the extent of injury. It involves the use of rigid and flexible bronchoscope and the capability to endotracheal intubation by fiber optic bronchoscope. Interventional pulmonologist should evaluate the distance from vocal cords and tracheal speron, dimension of the injury and bulging in the esophagus. ITI causes longitudinal tears of the posterior tracheal wall or the junction between cartilage and the posterior membrane on the right, and rarely extends to main bronchi. 74

Cardillo et al. proposed a morphologic classification of ITI (Table IV). ${ }^{75}$

ITI presenting with acute respiratory distress requires immediate action to promptly and effectively re-establish and secure a patient airway and relieve the injury. This is often achieved with rigid bronchoscope. Since these patients usually present in acute conditions, if there is any doubt regarding the stability of the airways, rigid bronchoscopy is the procedure of choice as it ensures a secure airway, enabling oxygenation and ventilation. It also serves as a therapeutic tool for severe tracheal injuries.

TABLE IV.-Tracheal Wall Injury: Morphologic Classification.

Level I Mucosal or submucosal injury without mediastinal emphysema and esophageal injury

Level II Lesion extending to the muscular wall with subcutaneous or mediastinal emphysema without esophageal injury or mediastinitis

Level IIIA Complete laceration with esophageal or mediastinal soft-tissue herniation without esophageal injury or mediastinitis

Level IIIB Any laceration with esophageal injury or mediastinitis

Modified with permission from Cardillo et al. ${ }^{60}$ 
The establishment of a secure airway may require endotracheal intubation or rigid bronchoscopy.

Skills

The Interventional Pulmonologist is pivotal in the diagnosis and urgent (often definitive) treatment of ITI (conservative treatment $v s$. surgery or stent) (Table V).

- Pathophysiology and radiographic interpretation of tracheal lesion (CT scan).

- Interpretation of endoscopic images of lesions and asses extent of injury.

- Initial stabilization and decision regarding urgency and emergency

- Decision regarding endoscopic or surgical approach depending on acuteness of presentation, underlying cause and type of lesion, patient stability

- Basic bronchoscopy and interventional procedures with rigid bronchoscopy

- Patients with high surgical risk can be treated by placement of a covered self expanding metallic stent (SEMS) or a silicone stent. It mechanically obstruct the tracheal defect and cause an exuberant inflammatory response with granulation tissue formation.

The stent can be removed after 4 to 6 weeks as the healing of the tear is noted. ${ }^{75}$

\section{Procedural steps}

1) Clinical suspicion (acute respiratory distress after extubation; pneumothorax, pneumomediastinum)

2) CT chest imaging (with oral contrast in case of concomitant esophageal injury)

3) Bronchoscopy (diagnosis and treatment)

- Reintubation under bronchoscopic guidance to place the ET cuff beyond the site of the injury, to avoid worsen-

TABLE V.-Indications for conservative and surgical treatment of tracheobronchial injury. ${ }^{75}$



Conservative treatment

Spontaneously breathing patients

Minimal mechanical ventilation

No evidence of esophageal or mediastinal compromise

Surgical indications after failure of conservative treatment

Worsening subcutaneous emphysema

Progressive pneumomediastinum

Pneumothorax with persistent air leak

Pneumothorax with failure of lung re-expansion despite chest tube placement Emergency surgical indications

Esophageal wall prolapses into the tracheobronchial lumen

Failure to ventilate

Intraoperative diagnosis of tracheobronchial injury ing of the tear and to prevent the formation of a false track.

- Selective intubation of the mainstem bronchus in case of tracheal tear extending to the carina

- Mechanical ventilatory support / single lung ventilation

- If intubated patient deflate the ET cuff, manipulate the cuff to visualize the tracheal injury

- assess the injury extent

4) Upper gastroenterologic endoscopy if complete laceration of the tracheal wall (to assess esophageal injury)

In case of concomitant esophageal injury

- Broad-spectrum prophylactic antibiotics for at least 1 week

- Temporary percutaneous gastrostomy tube for nutrition ( avoid nasogastric/orogastric feeding tube)

Theoretical and practical training: resources and assessment tools for "Core Competences"

As described above, ITI must to be treated by a very welltrained multidisciplinary specialist team. ${ }^{61}$ The rarity of these events does not make possible a specific and direct training, even in these emergency situations a consolidated set of skills with the fiberscope is essential. The training programs must provide a theoretical approach that allows: fast diagnostic classification (especially the assessment of the lesion); careful assessment of the clinical context; to establish conservative or surgical treatment.

Qualitative and quantitative assessment

General theoretical and practical training common to all procedures.

Specifically:

1) Live sessions and clinical case discussions, with emphasis on the strategy to be applied in different situations

2) Non-technical skill simulation as training on how to manage the complications within the multidisciplinary team, with anesthetist and nurses

3) Training on the patient with a tutor and then under supervision: a video monitor for larger image display may be most effective for the novice or trainee

4) Training on patient under supervision (in cases where it is allowed by law) $)^{55}$

Number of procedures: unknown since ITI is a lifethreatening scenario

Register all the procedures by log-book

Qualitative assessment is based on clinical outcome after ITI diagnosis and treatment. (improvement in symptoms, quality of life and survival) and complications. ${ }^{55}$ 


\section{References}

1. Fiadjoe J, Stricker P. Pediatric difficult airway management: current devices and techniques. Anesthesiol Clin 2009;27:185-95.

2. Foltran F, Ballali S, Passali FM, Kern E, Morra B, Passali GC, et al. Foreign bodies in the airways: a meta-analysis of published papers. Int $\mathrm{J}$ Pediatr Otorhinolaryngol 2012;76(Suppl 1):S12-9.

3. Casalini AG, Majori M, Anghinolfi M, Burlone E, D'Ippolito R, Toschi $\mathrm{M}$, et al. Foreign body aspiration in adults and in children: advantages and consequences of a dedicated protocol in our 30-year experience. J Bronchology Interv Pulmonol 2013;20:313-21.

4. Boufersaoui A, Smati L, Benhalla KN, Boukari R, Smail S, Anik K, et al. Foreign body aspiration in children: experience from 2624 patients. Int J Pediatr Otorhinolaryngol 2013;77:1683-8.

5. Tahir N, Ramsden WH, Stringer MD. Tracheobronchial anatomy and the distribution of inhaled foreign bodies in children. Eur J Pediatr 2009;168:289-95.

6. Donato L, Mai Hong Tran T, Ghori UK, Musani AI. Pediatric Interventional Pulmonology. Clin Chest Med 2018;39:229-38.

7. Mantor PC, Tuggle DW, Tunell WP. An appropriate negative bronchoscopy rate in suspected foreign body aspiration. Am J Surg 1989;158:622-4.

8. Martinot A, Closset M, Marquette CH, Hue V, Deschildre A, Ramon $\mathrm{P}$, et al. Indications for flexible versus rigid bronchoscopy in children with suspected foreign-body aspiration. Am J Respir Crit Care Med 1997;155:1676-9.

9. de Blic J, Delacourt C, Scheinmann P. Ultrathin flexible bronchoscopy in neonatal intensive care units. Arch Dis Child 1991;66:1383-5.

10. Hasegawa S, Hitomi S, Murakawa M, Mori K. Deyelopment of an ultrathin fiberscope with a built-in channel for bronchoscopy in infants. Chest 1996;110:1543-6.

11. Roberts S, Thornington RE. Paediatric bronchoscopy. Contin Educ Anaesth Crit Care Pain 2005;5:41-4.

12. Prakash UB, Midthun DE, Edell ES. Indications for flexible versus rigid bronchoscopy in children with suspected foreign-body aspiration. Am J Respir Crit Care Med 1997;156:1017-9.

13. Eber E, Antón-Pacheco JL, de Blic J, Doull I, Faro A, Nenna R, et al. ERS statement: interventional bronchoscopy in children. Eur Respir J 2017;50:1700901.

14. Farrell PT. Rigid bronchoscopy for foreign body removal: anaesthesia and ventilation. Paediatr Anaesth 2004;14:84-9.

15. Lee YJ, Lee SM, Park JS, Yim JJ, Yang SC, Kim YW, et al. The clinical implications of bronchoscopy in hemoptysis patients with no explainable lesions in computed tomography. Respir Med 2012;106:413-9.

16. Lordan JL, Gascoigne A, Corris PA. The pulmonary physician in critical care * Illustrative case 7 : assessment and management of massive haemoptysis. Thorax 2003;58:814-9.

17. Sakr L, Dutau H. Massive hemoptysis: an update on the role of bronchoscopy in diagnosis and management. Respiration 2010;80:38-58.

18. Amirana M, Frater R, Tirschwell P, Janis M, Bloomberg A, State D. An aggressive surgical approach to significant hemoptysis in patients with pulmonary tuberculosis. Am Rev Respir Dis 1968;97:187-92.

19. Crocco JA, Rooney JJ, Fankushen DS, DiBenedetto RJ, Lyons HA. Massive hemoptysis. Arch Intern Med 1968;121:495-8.

20. Corey R, Hla KM. Major and massive hemoptysis: reassessment of conservative management. Am J Med Sci 1987;294:301-9.

21. Hirshberg B, Biran I, Glazer M, Kramer MR. Hemoptysis: etiology, evaluation, and outcome in a tertiary referral hospital. Chest 1997;112:440-4

22. Flume PA, Yankaskas JR, Ebeling M, Hulsey T, Clark LL. Massive hemoptysis in cystic fibrosis. Chest 2005;128:729-38.

23. Ong TH, Eng P. Massive hemoptysis requiring intensive care. Intensive Care Med 2003;29:317-20.
24. Khalil A, Soussan M, Mangiapan G, Fartoukh M, Parrot A, Carette MF. Utility of high-resolution chest CT scan in the emergency management of haemoptysis in the intensive care unit: severity, localization and aetiology. Br J Radiol 2007;80:21-5.

25. Holsclaw DS, Grand RJ, Shwachman H. Massive hemoptysis in cystic fibrosis. J Pediatr 1970;76:829-38.

26. Remy J, Remy-Jardin M, Voisin C. Endovascular management of bronchial bleeding. In: Butler J, editor. The Bronchial Circulation. New York: Dekker; 1992. P. 667-723.

27. Yoon W, Kim JK, Kim YH, Chung TW, Kang HK. Bronchial and nonbronchial systemic artery embolization for life-threatening hemoptysis: a comprehensive review. Radiographics 2002;22:1395-409.

28. Fartoukh M, Khalil A, Louis L, Carette MF, Bazelly B, Cadranel J, et al. An integrated approach to diagnosis and management of severe haemoptysis in patients admitted to the intensive care unit: a case series from a referral centre. Respir Res 2007;8:11.

29. Panos RJ, Barr LF, Walsh TJ, Silverman HJ. Factors associated with fatal hemoptysis in cancer patients. Chest 1988;94:1008-13.

30. Shigemura N, Wan IY, Yu SC, Wong RH, Hsin MK, Thung HK, et al. Multidisciplinary management of life-threatening massive hemoptysis: a 10-year experience. Ann Thorac Surg 2009;87:849-53.

31. Thirumaran M, Sundar R, Sutcliffe IM, Currie DC. Is investigation of patients with haemoptysis and normal chest radiograph justified? Thorax 2009;64:854-6.

32. Revel MP, Fournier LS, Hennebicque AS, Cuenod CA, Meyer G, Reynaud P, et al. Can CT replace bronchoscopy in the detection of the site and cause of bleeding in patients with large or massive hemoptysis? AJR Am J Roentgenol 2002;179:1217-24.

33. Gasparini S. Indications for diagnostic bronchoscopy in adults. Monaldi Arch Chest Dis 2011;75:24-31.

34. Haponik EF, Fein A, Chin R. Managing life-threatening hemoptysis: has anything really changed? Chest 2000;118:1431-5.

35. Löschhorn C, Nierhoff N, Mayer R, Zaunbauer W, Neuweiler J, Knoblauch A. Dieulafoy's disease of the lung: a potential disaster for the bronchoscopist. Respiration 2006;73:562-5.

36. AIPO GS Endoscopia toracica. Standard operativi e linee guida in endoscopia toracica. Rassegna dell'Apparato Respiratorio; 1997 [Internet]. Available from: https://docplayer.it/13795447-Standard-operativi-e-lineeguida-in-endoscopia-toracica.html [cited 2018, Oct 17].

37. Douketis JD, Spyropoulos AC, Spencer FA, Mayr M, Jaffer AK, Eckman $\mathrm{MH}$, et al. Perioperative management of antithrombotic therapy: Antithrombotic Therapy and Prevention of Thrombosis, 9th ed: American College of Chest Physicians Evidence-Based Clinical Practice Guidelines. Chest 2012;141(Suppl):e326S-50S.

38. Daniels PR. Peri-procedural management of patients taking oral anticoagulants. BMJ 2015;351:h2391.

39. Bernasconi M, Koegelenberg CF, Koutsokera A, Ogna A, CasuttA, Nicod $\mathrm{L}$, et al. Iatrogenic bleeding during flexible bronchoscopy: risk factors, prophylactic measures and management. ERJ Open Res 2017;3:00084-02016.

40. Dweik RA, Stoller JK. Role of bronchoscopy in massive hemoptysis. Clin Chest Med 1999;20:89-105.

41. Conlan AA, Hurwitz SS. Management of massive haemoptysis with the rigid bronchoscope and cold saline lavage. Thorax 1980;35:901-4.

42. Kalyanaraman M, Carpenter RL, McGlew MJ, Guertin SR. Cardiopulmonary compromise after use of topical and submucosal alpha-agonists: possible added complication by the use of beta-blocker therapy. Otolaryngol Head Neck Surg 1997;117:56-61.

43. Breuer HW, Charchut $\mathrm{S}$, Worth $\mathrm{H}$, Trampisch HJ, Glänzer K. Endobronchial versus intravenous application of the vasopressin derivative glypressin during diagnostic bronchoscopy. Eur Respir J 1989;2:225-8.

44. Tüller C, Tüller D, Tamm M, Brutsche MH. Hemodynamic effects of endobronchial application of ornipressin versus terlipressin. Respiration 2004;71:397-401. 
45. Tscheikuna J, Chvaychoo B, Naruman C, Maranetra N. Tranexamic acid in patients with hemoptysis. J Med Assoc Thai 2002;85:399-404.

46. Bellam BL, Dhibar DP, Suri V, Sharma N, Varma SC, Malhotra S, et al. Efficacy of tranexamic acid in haemoptysis: A randomized, controlled pilot study. Pulm Pharmacol Ther 2016;40:80-3.

47. Tsukamoto T, Sasaki H, Nakamura H. Treatment of hemoptysis patients by thrombin and fibrinogen-thrombin infusion therapy using a fiberoptic bronchoscope. Chest 1989;96:473-6.

48. de Gracia J, de la Rosa D, Catalán E, Alvarez A, Bravo C, Morell F. Use of endoscopic fibrinogen-thrombin in the treatment of severe hemoptysis. Respir Med 2003;97:790-5.

49. Valipour A, Kreuzer A, Koller H, Koessler W, Burghuber OC. Bronchoscopy-guided topical hemostatic tamponade therapy for the management of life-threatening hemoptysis. Chest 2005;127:2113-8.

50. Bhattacharyya P, Dutta A, Samanta AN, Chowdhury SR. New procedure: bronchoscopic endobronchial sealing; a new mode of managing hemoptysis. Chest 2002;121:2066-9.

51. Chawla RK, Madan A, Aditya C. Glue in Hemoptysis. J Bronchology Interv Pulmonol 2016;23:e40-2.

52. Freitag L, Tekolf E, Stamatis G, Montag M, Greschuchna D. Three years experience with a new balloon catheter for the management of haemoptysis. Eur Respir J 1994;7:2033-7.

53. Kho SS, Chan SK, Yong MC, Tie ST. Endobronchial embolization for life-threatening hemoptysis with Endobronchial Watanabe Spigot. BMC Res Notes 2017;10:304.

54. Ernst A, Silvestri GA, Johnstone D; American College of Chest Physicians. Interventional pulmonary procedures: Guidelines from the American College of Chest Physicians. Chest 2003;123:1693-717.

55. Corbetta L, Patelli M. Executive summary of training and competence standards for the interventional pulmonology master program in Italy. J Bronchology Interv Pulmonol 2018;25:6-8.

56. Ernst A, Wahidi MM, Read CA, Buckley JD, Addrizzo-Harris DJ, Shah PL, et al. Adult Bronchoscopy Training: Current State and Suggestions for the Future: CHEST Expert Panel Report. Chest 2015;148:321-32.

57. Prokakis C, Koletsis EN, Dedeilias P, Fligou F, Filos K, Dougenis D. Airway trauma: a review on epidemiology, mechanisms of injury, diagnosis and treatment. J Cardiothorac Surg 2014;9:117.

58. Gómez-Caro Andrés A, Moradiellos Díez FJ, Ausín Herrero P, DíazHellín Gude V, Larrú Cabrero E, de Miguel Porch E, et al. Successful conservative management in iatrogenic tracheobronchial injury. Ann Thorac Surg 2005;79:1872-8.

59. Panagiotopoulos N, Patrini D, Barnard M, Koletsis E, Dougenis D, Lawrence D. Conservative versus Surgical Management of Iatrogenic Tracheal Rupture. Med Princ Pract 2017;26:218-20.
60. Cardillo G, Carbone L, Carleo F, Batzella S, Jacono RD, Lucantoni $\mathrm{G}$, et al. Tracheal lacerations after endotracheal intubation: a proposed morphological classification to guide non-surgical treatment. Eur J Cardiothorac Surg 2010;37:581-7.

61. Frova G, Sorbello M. Iatrogenic tracheobronchial ruptures: the debate continues. Minerva Anestesiol 2011;77:1130-3.

62. Miñambres E, Burón J, Ballesteros MA, Llorca J, Muñoz P, GonzálezCastro A. Tracheal rupture after endotracheal intubation: a literature systematic review. Eur J Cardiothorac Surg 2009;35:1056-62.

63. Kiser AC, O'Brien SM, Detterbeck FC. Blunt tracheobronchial injuries: treatment and outcomes. Ann Thorac Surg 2001;71:2059-65.

64. Deja M, Menk M, Heidenhain C, Spies CD, Heymann A, Weidemann $\mathrm{H}$, et al. Strategies for diagnosis and treatment of iatrogenic tracheal ruptures. Minerva Anestesiol 2011;77:1155-66.

65. Bonniot JP, Even P. Partial tracheal rupture after intubation: surgical abstention and endoscopy surveillance. Nouv Presse Med 1979;8:781. French.

66. d'Odemont JP, Pringot J, Goncette L, Goenen M, Rodenstein DO. Spontaneous favorable outcome of tracheal laceration. Chest 1991;99:1290-2.

67. Mitchell JB, Ward PM. The management of tracheal rupture using bilateral bronchial intubation. Anaesthesia 1993;48:223-5.

68. Ross HM, Grant FJ, Wilson RS, Burt ME. Nonoperative management of tracheal laceration during endotracheal intubation. Ann Thorac Surg $1997 ; 63: 240-2$

69. Marquette $\mathrm{CH}$, Bocquillon $\mathrm{N}$, Roumilhac $\mathrm{D}$, Nevière R, Mathieu D, Ramon P. Conservative treatment of tracheal rupture. J Thorac Cardiovasc Surg 1999;117:399-401.

70. Kaloud H, Smolle-Juettner FM, Prause G, List WF. Iatrogenic ruptures of the tracheobronchial tree. Chest 1997;112:774-8.

71. Jougon J, Ballester M, Choukroun E, Dubrez J, Reboul G, Velly JF. Conservative treatment for postintubation tracheobronchial rupture. Ann Thorac Surg 2000;69:216-20.

72. Conti M, Pougeoise M, Wurtz A, Porte H, Fourrier F, Ramon P, et al. Management of postintubation tracheobronchial ruptures. Chest 2006;130:412-8.

73. Gómez-Caro A, Ausín P, Moradiellos FJ, Díaz-Hellín V, Larrú E, Pérez JA, et al. Role of conservative medical management of tracheobronchial injuries. J Trauma 2006;61:1426-34, discussion 1434-5.

74. Grillo HC. Repair of tracheobronchial trauma. In: Grillo HC, editor. Surgery of the trachea and bronchi. Hamilton, ON, Canada: BC Decker Inc; 2004. p. 632.

75. Grewal HS, Dangayach NS, Ahmad U, Ghosh S, Gildea T, Mehta AC Treatment of tracheobronchial Injuries. A contemporary Review. Chest 2018 Jul 27. [Epub ahead of print]

Conflicts of interest.-The authors certify that there is no conflict of interest with any financial organization regarding the material discussed in the manuscript. Article first published online: October 5, 2018. - Manuscript accepted: September 17, 2018. - Manuscript received: September 6, 2018. 